\title{
Tecnologias Móveis em Contexto Educativo: uma Revisão Sistemática da Literatura
}

Ernane Rosa Martins, IFG, Brasil, ernane.martins@ifg.edu.br

Wendell Bento Geraldes, IFG, Brasil, wendell.geraldes@ifg.edu.br

Ulisses Rodrigues Afonseca, IFG, Brasil, urafonseca@ifg.edu.br

Luís Manuel Borges Gouveia, UFP, Portugal, lmbg@ufp.edu.pt

Resumo. Este artigo apresenta uma revisão sistemática da literatura sobre as produções disponíveis em algumas bases de dados acerca das Tecnologias Móveis em contexto educativo. Neste sentido, foram analisados 174 trabalhos que se encontravam disponíveis online e que apresentavam estudos empíricos ou teóricos sobre a aplicabilidade deste tipo de tecnologias no processo de ensino e aprendizagem. Os resultados mostram de que forma as tecnologias móveis têm sido utilizada em contexto educativo, assim como, identificou os benefícios e dificuldades no uso dos dispositivos móveis, possíveis estratégias para aprimorar as práticas docentes com o uso de tecnologias móveis em sala de aula e o que ainda falta ser explorado sobre este tema.

Palavras-chave: Revisão Sistemática, Tecnologias Móveis, Ensino, Aprendizagem.

\section{Mobile Technologies in Educational Context: a Systematic Review of Literature}

Abstract. This article presents a systematic review of the literature on the productions available in some databases on Mobile Technologies in an educational context. In this sense, we analyzed 174 works that were available online and that presented empirical or theoretical studies on the applicability of this type of technologies in the process of teaching and learning. The results show how mobile technologies have been used in educational context, as well as, identified the benefits and difficulties in the use of mobile devices, possible strategies to improve teaching practices with the use of mobile technologies in the classroom and what still need to be explored on this topic.

Keywords: Systematic Review, Mobile Technologies, Teaching, Learning.

\section{Introdução}

A nossa era é conhecida como a era do conhecimento, visto a importância dada hoje ao conhecimento, em todos os setores, sobretudo em consequência da informatização e do processo de globalização das telecomunicações. Todavia, o que vemos é a predominância da difusão de dados e informações e não de conhecimentos. Isto é possível graças às novas tecnologias que estocam o conhecimento, de forma prática e acessível, em gigantescos volumes de informações, permitindo a pesquisa e o acesso de maneira muito simples, amigável e flexível. Não somente o aceso, mas também como emissor de informações. Pela internet, a partir de qualquer lugar e a qualquer hora, pode-se acessar inúmeras bibliotecas em muitas partes do mundo, permitindo acessar conhecimentos não apenas por palavras, mas também por imagens, sons, fotos, vídeos, etc (Gadotti, 2000).

As tecnologias de comunicação modificam algumas das funções dos professores, mas não os substituem. A tarefa de transmitir informações, passa a ser feita por bancos de dados, livros, vídeos, programas em $\mathrm{CD}$, os professores agora tem o papel de estimulador da curiosidade do aluno na busca de querer conhecer, pesquisar e buscar as informações mais relevantes. É ele que coordena o processo de apresentação dos resultados pelos alunos, contextualiza os resultados, os adapta à realidade dos alunos e 
questiona os dados apresentados, também transforma informação em conhecimento e conhecimento em saber, em vida e em sabedoria (Moran, 1995).

As tecnologias permitem um novo encantamento na escola, abrindo suas paredes e possibilitando que alunos conversem e pesquisem com outros alunos da mesma cidade, país ou do exterior, no seu próprio ritmo. Acontecendo o mesmo com os professores. Seus trabalhos de pesquisa podem ser compartilhados por outros alunos e divulgados instantaneamente na rede. Encontram-se disponíveis para alunos e professores inúmeras bibliotecas eletrônicas, revistas on line, textos, imagens e sons, que facilitam a tarefa de preparar as aulas, fazer trabalhos de pesquisa e ter materiais atraentes para apresentação. O professor passa a estar mais próximo do aluno, podendo receber mensagens com dúvidas, passar informações complementares para determinados alunos, adaptar a sua aula para o ritmo de cada aluno, procurar ajuda em outros colegas sobre problemas que surgem, novos programas para a sua área de conhecimento. $\mathrm{O}$ processo de ensino-aprendizagem pode ganhar mais dinamismo, inovação e poder de comunicação inusitados (Moran, 1995).

O mundo atualmente exige um profissional cada vez mais crítico, criativo, com capacidade de pensar, de aprender a aprender, de trabalhar em grupo e de conhecer o seu potencial intelectual, com capacidade de constante aprimoramento e depuração de ideias e ações. Essa nova atitude não é passível de ser transmitida mas deve ser construída e desenvolvida por cada indivíduo, ou seja, deve ser fruto de um processo educacional em que o aluno vivencie situações que lhe permitam construir e desenvolver essas competências. Neste contexto o computador e as novas tecnologias podem ser importantes aliados neste processo (Valente, 2012). Pensando nas possíveis publicações advindas da possibilidade pedagógica do uso das tecnologias móveis, é que se decidiu sistematizar um estudo a fim de mapear e conhecer o que os pesquisadores têm descoberto quando utilizam estas tecnologias com seus alunos. Por esta razão, a questão problema norteadora que direcionou a realização desde estudo foi: "De que forma as tecnologias móveis têm sido utilizada em contexto educativo?". Além desta pergunta central, estabeleceu-se outras perguntas complementares. Quais os benefícios apresentados? Quais as dificuldades encontradas? Quais as possíveis estratégias para tornar o professor melhor preparado? O que ainda falta ser explorado?

$\mathrm{O}$ estudo justifica-se na medida em que, hoje em dia, grande parte dos alunos e professores, possuem aparelho celular e, praticamente todos, são smartphones com acesso à internet. Assim como, a constatação que estes possuem grandes potencialidades e possibilidades de utilização no ensino de todas as áreas do conhecimento. O que pode proporcionar ricas experiências ao professor e aos alunos, assim como, um maior compartilhamento de informações. Este artigo está estruturado da seguinte forma. Além desta introdução, na seção 2, é apresentada uma revisão bibliográfica. Na seção 3 é descrita a metodologia utilizada na pesquisa. A seção 4 relata os resultados alcançados e discussões. Por fim, na seção 5 são apresentadas as considerações finais e as perspectivas de trabalhos futuros.

\section{Revisão Bibliográfica}

A popularização da internet e de suas mídias mudou o cenário educacional brasileiro, por meio da utilização dos meios de comunicação virtual, digital e dos dispositivos móveis na escola, ou seja, estes passaram a integrar-se como instrumentos pedagógicos para a aquisição do conhecimento. Por isto, o professor se depara com o desafio de acompanhar esse ritmo, buscando integrar a cultura extraescolar dos alunos (Sena \& Burgos, 2010). Wains \& Mahmood (2008) definem o M-learning (mobile 
learning - aprendizagem por meio de dispositivos móveis) com sendo "um campo emergente, que engloba tecnologias sem fio e computação móvel para permitir que a aprendizagem possa ocorrer em qualquer tempo e em qualquer lugar, maximizando a liberdade dos alunos". O Mobile Learning ou m-Learning surgiu a partir da disponibilidade de dispositivos móveis e considerando as necessidades específicas da educação e treinamentos (Nyiri, 2002).

As inovações tecnológicas provenientes do desenvolvimento das telecomunicações estão oportunizando acesso a diferentes ambientes e formas de aprendizagem. Anteriormente precisava-se de um aparelho ligado a uma estrutura fixa de rede, atualmente os dispositivos móveis permitem o acesso a ambientes e recursos educacionais similares (Mülbert \& Pereira, 2011). O telefone celular é o mais popular e acessível dos dispositivos que podem suportar o Mobile Learning, não necessitando de investimentos financeiros por parte das instituições, por se tratar de uma ferramenta comum e disponível no cotidiano dos alunos "Se o computador ainda é um objeto restrito, o celular está presente em boa parte das escolas, nas mochilas dos alunos de diferentes classes sociais" (Merije, 2012, p.81). Os smartphones é uma tecnologia que reúne várias mídias num só aparelho (telefone, internet, console de jogos, recursos dos computadores pessoais, etc.). A associação dos recursos dos aparelhos celulares e das redes de telefonia móvel com os da internet possibilitou o acesso e compartilhamento de conteúdo, o que proporcionou uma nova dinâmica aos processos de comunicação, assim como, a aprendizagem (Merije, 2012). A revolução digital, a miniaturização de aparelhos e a conectividade com redes de comunicação, possibilitaram misturar o digital com o físico, criando um ambiente de tecnologia semântica e cognitiva, que vem remodelando as formas de fazer, criar, pensar e relacionar a vida cotidiana, ao trabalho, ao lar, ao lazer, a educação ou a qualquer outro espaço (Cordeiro \& Bonilla, 2015).

O m-Learning conforme Marçal et al. (2005), surge como uma importante alternativa de ensino e treinamento à distância, na qual podem ser destacados os seguintes objetivos: Melhorar os recursos para o aprendizado do aluno, que poderá contar com um dispositivo computacional para execução de tarefas, anotação de ideias, consulta de informações via internet, registro de fatos através de câmera digital, gravação de sons e outras funcionalidades existentes; Prover acesso aos conteúdos didáticos em qualquer lugar e a qualquer momento, de acordo com a conectividade do dispositivo; Aumentar as possibilidades de acesso ao conteúdo, incrementando e incentivando a utilização dos serviços providos pela instituição, educacional ou empresarial; Expandir o corpo de professores e as estratégias de aprendizado disponíveis, através de novas tecnologias que dão suporte tanto à aprendizagem formal como à informal; Fornecer meios para o desenvolvimento de métodos inovadores de ensino e de treinamento, utilizando os novos recursos de computação e de mobilidade.

Segundo o relatório da UNESCO intitulado "O Futuro da Aprendizagem Móvel”, de 2014, a aprendizagem móvel por meio da EaD se apresenta como área de foco, já que a cada dia mais o uso de dispositivos móveis nos sistemas formais de educação se assume como um modelo popular de estudos e de pesquisas no mundo. Existe pelo menos dois modelos principais de uso da Tecnologia Móvel: Um Computador por Aluno (UCA), em que cada aluno utiliza um computador para uso escolar, e Bring Your Own Device (BYOD), em que cada aluno utiliza seu próprio aparelho móvel. O modelo BYOD, vem causando mudanças sem precedentes na educação superior e no ensino a distância permitindo que cada vez mais alunos acessem os diferentes materiais pedagógicos através da tecnologia móvel. Aproveitando este aumento do número de pessoas que têm acesso ou possui um dispositivo móvel, as 
iniciativas BYOD apresentam-se como promissoras para alunos de todos os cantos do mundo (Unesco, 2014).

Nos cursos superiores o uso dos dispositivos móveis, tais como: smartphones e tablets estão colaborando para a leitura de textos em aplicativos específicos, substituindo os papéis pela tela digital e diminuindo a quantidade de livros e cadernos. Também disponibilizam bate-papos através de aplicativos de comunicação, tais como: WhatsApp e Messenger, possibilitando chats instantâneos individuais ou em grupo entre professores e alunos, servindo de apoio para minimizar as dificuldades. Outra questão importante é a possibilidade de oferecer um espaço digital, onde professor e alunos podem acessar o tempo que quiserem e na hora mais conveniente, podendo ler textos, ouvir e/ou assistir os recursos das aulas, se comunicar instantaneamente ou publicar seu trabalho em qualquer momento, desde que haja conexão com à internet (Boll; Lopes \& Luchini, 2016). É notório a importância da inserção de novos instrumentos no contexto escolar, todavia, a mobilidade, característica essencial dos dispositivos moveis, permite serem utilizados em ambientes também fora das salas de aula. Assim, fica visível a necessidade de encorajarmos o processo de aprendizagem para além dos parâmetros tradicionais, propiciando apropriação de conhecimentos também em ambientes extraescolares (Santana, 2016).

$\mathrm{O}$ acesso às tecnologias digitais podem possibilitar a descentralização do conhecimento, a diversidade e a promoção da colaboração entre sujeitos produtores de cultura e da transformação social (Knobel \& Lankshear, 2007). A mobilidade comunicacional transcende tempo e espaço, o acesso ao conhecimento está cada vez mais relacionado ao espaço conectivo da rede comunicativa, dependendo da mobilidade da cultura digital. Assim, o conceito de aprendizagem móvel relaciona-se com a ampliação das possibilidades do processo comunicativo entre alunos conectados e professor (Lemos \& Josgrilberg, 2009). As tecnologias móveis se tornaram parte da vida das crianças em todo o mundo, com isto muitos governos e escolas estão utilizando estes dispositivos para uma série de diferentes finalidades de ensino e aprendizagem, e promovendo diversos estudos nesta área, demostrando que estes ajudam a promover a aprendizagem, competências e perspectivas que as crianças necessitam para competir e cooperar atualmente. Centrando no desenvolvimento de competências, tais como: colaboração, pensamento crítico e resolução de problemas. Alguns projetos exploram as funcionalidades inovadoras dos dispositivos móveis, outros contam apenas com as funcionalidades standard, outros as capacidades de personalização, e outros procuram saber como os dispositivos móveis podem encorajar a colaboração em trabalho de equipe (Moura, 2009).

Inúmeros os novos desafios pedagógicos apresentados pela utilização e integração das Tecnologias da Informação e da Comunicação (TIC), obrigando à redefinirmos os papéis dos diferentes parceiros no processo educativo. Assim, as TIC podem ser apresentadas como um reforço aos métodos tradicionais de ensino ou como uma forma de renovação das oportunidades de aprendizagem (Barros, 2011). Alguns requisitos são indispensáveis para que as tecnologias se tornem atrativas para o usuário. Esses requisitos, conforme Lee et al. (2004), são: Mobilidade, capacidade de deslocamento do dispositivo em qualquer hora e qualquer lugar; Portabilidade, facilidade de se transportar os dispositivos, devendo ser geometricamente confortáveis e leves; Usabilidade, utilidade funcional do dispositivo, que deve ter capacidade de uso e flexibilidade para qualquer tipo de indivíduo, principalmente para portadores de necessidades especiais; Funcionalidade, oferecer inúmeras opções de uso, de forma a atender às demandas do usuários. Segundo os mesmos autores estes requisitos atraem 
como principais públicos-alvo os jovens e adultos de gerações mais recentes. Usuários com esse perfil encontram nessas novas tecnologias (SMS, Facebook, Skype, Whatsapp etc.), a possibilidade de entretenimento e educação juntas, por meio da comunicação textual.

As tecnologias não são transformadoras dos contextos educacionais. Simplesmente inseri-las na educação, sem criar uma proposta didático-pedagógica consistente, coerente e com um planejamento alinhado com as necessidades dos alunos, não permite explorar suas potencialidades e possibilitar o desenvolvimento de práticas educacionais diferenciadas. São várias as potencialidades, mas as tecnologias apenas serão bem utilizadas no contexto educacional se as propostas metodológicas forem suficientemente abertas, criativas e focadas no aprendiz (Costa; Silva; Cordeiro; Silva, 2014, p. 59). Segundo Lucena (2016, p. 287) as tecnologias aplicadas no âmbito educacional atuam como ferramentas pedagógicas que "potencializa a produção de saberes construídos de forma coletiva e colaborativa, utilizando as redes sociodigitais".

\section{Metodologia}

Para a realização desta pesquisa utilizou-se como procedimento uma revisão sistemática da literatura, que é uma metodologia eficaz para integrar as informações de um conjunto de trabalhos realizados separadamente, ou seja, investigar o atual estado da arte. Esses trabalhos podem apresentar resultados conflitantes e/ou coincidentes, bem como, identificar novos temas que necessitem de uma pesquisa realizada dentro de preceitos científicos, de modo a conhecer melhor, auxiliando na orientação para possíveis investigações futuras (Sampaio \& Mancini, 2007). Para Gonçalves, Nascimento \& Nascimento (2015, p. 194), a revisão sistemática da literatura "possibilita uma investigação que visa identificar evidências relacionadas a um problema específico de pesquisa, com o intuito de destacar ideias, posturas e opiniões de autores, publicadas na área de conhecimento em que se insere".

Segundo Gonçalves, Nascimento \& Nascimento (2015, p.195) as principais etapas para a elaboração de uma revisão sistemática são: Problema de Pesquisa (questão a ser investigada); Protocolo de Pesquisa (descrição criteriosa do estudo); Bases de Dados (localização dos estudos); Critérios de Inclusão/Exclusão (características e especificidades dos estudos); Análise, Crítica e Avaliação (validade dos estudos selecionados); Elaboração do Resumo (Síntese dos conteúdos abordados); Identificação das Evidências (estudos agrupados conforme a semelhança); Conclusão (alcance das evidências identificadas). Assim, para selecionar a amostra utilizada, foram definidos alguns parâmetros de inclusão e exclusão. Este procedimento teve como objetivo auxiliar na composição de um conjunto de artigos que abordem o mesmo tema. Os fatores utilizados neste estudo podem ser visualizados na Tabela 1.

Tabela 1 - Fatores de inclusão e exclusão dos trabalhos encontrados.

\section{Fatores de Inclusão}

Artigos disponíveis no Google, Google Acadêmico, Periódicos Capes e Scielo;

Estudos sobre Tecnologias Móveis em contexto educacional;

Artigos empíricos ou teóricos. Artigos que abordam o processo de ensino aprendizagem através de aprendizagem móvel

\section{Fatores de Exclusão}

Artigos em outras bases de dados que não sejam Google, Google Acadêmico Periódicos Capes e Scielo;

Estudos sobre Tecnologias Móveis em outros contextos, que não sejam educacionais. Artigos sem enfoque no ensino ou na aprendizagem

Para a formação da base de dados documental dos artigos que compuseram a amostra deste estudo, executou-se uma busca sistemática na web nos motores de busca 
Google, Google Acadêmico, Periódicos Capes e Scielo. Para a busca dos artigos nas bases de dados, utilizou-se como descritores as seguintes palavras: "Tecnologias Móveis", "Tecnologias Móveis e Educação", "Tecnologias Móveis e Ensino" e "Tecnologias Móveis e sala de aula". Na busca dos artigos para realização da pesquisa, após a exclusão dos artigos em duplicidades e que atendiam aos critérios estabelecidos, encontrou-se um total de 174 artigos. Que em seguida foram exportados para o software ATLAS.ti, sendo analisados, codificados e categorizados. Os motores de busca e descritores, foram escolhidos a critério dos pesquisadores, mas serão complementadas com novas pesquisas.

\section{Resultados e Discussão}

Com a realização das codificações no software ATLAS.ti dos artigos foi possível encontrar os possíveis benefícios da utilização de tecnologias moveis na educação, ilustrados na Tabela 2.

Tabela 2 - Apresenta os possíveis benefícios da utilização de tecnologias moveis na educação.

\begin{tabular}{|c|}
\hline Benefícios da utilização de tecnologias moveis na educação \\
\hline Abandona o sistema de massa e adota um sistema interativo \\
\hline Estimula os alunos a fazerem descobertas sozinhos \\
\hline Insere os alunos em um processo de pesquisa \\
\hline Estimula os alunos a colaborar entre si \\
\hline Flexibiliza quando estudar, onde e por quanto tempo \\
\hline Transfere as tarefas para além dos ambientes físicos da sala de aula \\
\hline Amplia as experiências de aprendizagem dentro e fora da sala de aula \\
\hline Substitui a necessidade de um computador para acessar a internet em sala de aula \\
\hline Facilita a comunicação e troca de informações a qualquer hora e lugar \\
\hline Permitir a socialização e inclusão de pessoas com deficiência ou restrição de locomoção \\
\hline Possibilita a utilização de recursos de vídeo e áudio \\
\hline Fornece um estilo de educação com foco nas demandas e necessidades individuais de aprendizado \\
\hline
\end{tabular}

Para Feenberg (2010) o conceito de educação centrado na interatividade humana, facilita a participação de grupos menos favorecidos, podendo aumentar o nível cultural da população como um todo. Feitosa \& Machado (2014) apresentam um caso em Vitória, no Espírito Santo, Brasil, em que uma escola recolhe cerca de 400 celulares dos alunos todos os dias na entrada, devolvendo-os na saída. O motivo apresentado é que os celulares atrapalham o rendimento dos outros alunos e dos professores. Segundo a escola sem a utilização dos celulares o trabalho dos professores e a participação dos alunos melhorou. Alguns Estados como o do Paraná tem a Lei Estadual no 18.118/2014PR, de 24 de junho de 2014, que proíbe o uso de aparelhos/equipamentos eletrônicos em salas de aula para fins não pedagógicos, no ensino fundamental e médio, sendo permitido somente para fins pedagógicos, sob orientação e supervisão do profissional de ensino. Já o Estado de Santa Catarina também dispõe da Lei Estadual 14.363 que diz em seu artigo 1 (Santa Catarina, 2008): "Fica proibido o uso de telefone celular nas salas de aula das escolas públicas e privadas no Estado de Santa Catarina”.

Pompeo (2014) explica que proibir o uso de aparelhos eletrônicos é autoritário e retrógrado, o melhor jeito é adaptar-se à realidade e usar a tecnologia em favor da aula. O estudo de Beland \& Murphy (2015), investigou o impacto de restringir o uso de dispositivos móveis nas escolas em relação a produtividade do aluno, a pesquisa foi realizada com 91 escolas de ensino médio, em quatro grandes cidades da Inglaterra, e os resultados indicaram que há uma melhoria no desempenho dos alunos de apenas $6,41 \%$ nas escolas que introduziram uma proibição. E Guenaga et al. (2012) reforça que em vez 
de proibir o uso dos dispositivos móveis na educação, o melhor é criar meios que possibilitem a exploração de seus recursos.

Para concretizar os benefícios da aprendizagem móvel, a Organização das Nações Unidas para a Educação, a Ciência e a Cultura (2014), recomenda aos formuladores de políticas que desenvolvam ações, tais como: Criar ou atualizar as políticas referentes à aprendizagem móvel; formar professores sobre como fazer avançar a aprendizagem por meio de tecnologias móveis; Fornecer apoio e formação a professores por meio de tecnologias móveis; Criar e aperfeiçoar conteúdos educacionais para uso em aparelhos móveis; Ampliar e melhorar as opções de conectividade; Desenvolver estratégias para fornecer acesso igual a todos; Promover o uso seguro, responsável e saudável das tecnologias móveis; Usar as tecnologias móveis para melhorar a comunicação e a gestão educacional; Aumentar a conscientização sobre a aprendizagem móvel. A Tabela 3 apresenta as dificuldades encontradas na utilização de tecnologias moveis na educação.

Tabela 3 - Apresenta as possíveis dificuldades na utilização de tecnologias moveis na educação. Dificuldades na utilização de tecnologias moveis na educação

Não ter certeza se está correta e suspeitar da validade e da precisão da fonte de informação pesquisada O aluno ser incapaz de obter respostas ou solução para suas perguntas ou dúvidas Atrapalhar o rendimento dos outros alunos e dos professores Desvio de atenção e distração Cansaço na utilização por muito tempo Falta de bateria e banda larga suficiente

Despreparo dos professores, falta de capacidade para incorporar o uso de tecnologias móveis na sala de aula Falta de planejamento

Falta de ações bem financiadas, coerentes, de grande escala e altamente visíveis

Assegurar que os projetos levem em conta as realidades e limitações das infraestruturas que já existem para a educação e as TICs, bem como os contextos sociais e culturais dos distintos países e regiões, Colas em provas

Segundo Bedi (2014), o papel do professor vai além de simplesmente prover novas informações, mas também em ajudar os alunos a engajar-se no processo de abstração e diferenciação de informações importantes e confiáveis. A Tabela 4 apresenta possíveis estratégias para tornar o professor melhor preparado para a utilização de tecnologias móveis.

Tabela 4 - Apresenta as possíveis estratégias para tornar o professor melhor preparado para a utilização de tecnologias móveis.

Estratégias para tornar o professor melhor preparado para a utilização de tecnologias móveis Não se deve jogar a tecnologia na sala de aula esperando bons resultados, o professor precisa concentrarse na pedagogia, incrementando o uso da tecnologia

Reduzir as aulas expositivas, pois o ensino em massa não funciona para essa geração

Dar aos alunos o poder para colaborar, estimulando-os a trabalhar juntos e mostrar como acessar os especialistas em um determinado assunto disponível na Internet

Concentrar-se no aprendizado para a vida, e não apenas para uma prova, ou seja, ensinar como aprender e não o que saber

Usar a tecnologia para conhecer cada aluno, construindo programas de aprendizado apropriados para cada um

Criar programas educacionais com opções de customização, transparência, integridade, colaboração, diversão, velocidade e inovação nas experiências de aprendizado, baseando em projetos

O professor precisa reconhecer seus próprios hábitos digitais e usar suas próprias ferramentas digitais em suas vidas diárias 
Conforme Tapscott (2010, p. 180), para reduzir as aulas expositivas, pode-se iniciar fazendo perguntas aos alunos e ouvindo suas respostas. Também pode escutar as perguntas dos alunos e deixá-los descobrir a resposta, criando assim, junto com o professor, uma experiência de aprendizado. Para Batchelor, Herselman \& Traxler (2010) os educadores são fundamentais para incorporar as tecnologias no ensino e na aprendizagem. Castells (2003) diz que antes de mudar a tecnologia, reconstruir as escolas, reciclar os professores, é necessário criar uma nova pedagogia, baseada na interatividade, na personalização e no desenvolvimento da capacidade autônoma de aprender a pensar. Royle, Stager \& Traxler (2014), afirmam que os professores precisam primeiro reconhecer seus próprios hábitos digitais e usar suas próprias ferramentas digitais em suas vidas diárias, para depois usar essas ferramentas para fins de aprendizagem. Observou-se que o tema está sendo mais abordado pelas áreas tecnológicas do que as sociais e humanísticas. Observou-se também que as publicações estavam associadas a mais de uma área de conhecimento, por causa da natureza multidisciplinar do tema. A Tabela 5 apresenta o que ainda falta ser melhor explorado sobre as tecnologias móveis.

Tabela 5 - Apresenta o que ainda falta ser melhor explorado sobre as tecnologias móveis

\begin{tabular}{|c|}
\hline O que ainda falta ser melhor explorado \\
\hline Encontrar uma compreensão mais precisa sobre os atributos da computação sem fio que atendam a \\
requisitos educacionais
\end{tabular}

Reis et. al. (2017) realizaram uma revisão sistemática intitulada "Apropriação das Tecnologias da Informação e Comunicação no ensino de ciências: uma revisão sistemática da última década (2007-2016)", em que evidenciaram que "o professor tem um papel fundamental na utilização das TIC e, se esse uso for feito de modo despreocupado e simplista, não trará grandes mudanças”.

\section{Considerações Finais}

$\mathrm{Na}$ busca por responder os questionamentos desta pesquisa, foi realizada uma revisão sistemática interdisciplinar que identificou que a aprendizagem móvel (Mlearning) é um campo de pesquisa emergente e em expansão, devido à crescente mobilidade da sociedade atual. Sendo assim, a maioria dos alunos e professores estão fazendo uso dos dispositivos móveis de comunicação. Este uso envolve o acesso a materiais didáticos, acesso a ambientes de interação entre alunos e professor, acesso à atividades escolares e compartilhamento de arquivos. Em vários estudos verificou-se que os dispositivos móveis de comunicação apresentam-se como uma alternativa para ampliar as possibilidades dos estudantes, proporcionando novas formas para construção e desenvolvimento do conhecimento.

Entre tanto, para que se tenha êxito na condução das atividades que envolvam tecnologias móveis percebe-se que é importante que os professores estejam devidamente capacitados e aptos a elaborar atividades dinâmicas e motivadoras, fazendo com que os dispositivos móveis de comunicação, sejam ferramentas úteis e inovadoras no processo 
de ensino e aprendizagem, e não simplesmente meros reprodutores de conteúdo. Este estudo também conseguiu identificar e apresentar os benefícios e dificuldades no uso dos dispositivos móveis, possíveis estratégias para tornar o professor melhor preparado para a utilização das tecnologias móveis e o que ainda falta ser explorado sobre o tema.

Assim, foi possível verificar algumas das dificuldade encontradas na utilização de equipamentos para finalidades educacionais, como por exemplo, serem elementos distraidores e que perturbam a concentração dos alunos nas tarefas escolares, ou os enormes benefícios para a educação que a utilização dos dispositivos móveis pode trazer, como por exemplo, permitir aos alunos aprender em qualquer hora e lugar ou permitir a socialização e inclusão de pessoas com deficiência ou restrição de locomoção, se adaptando ao cotidiano das pessoas. Conforme o relatório da Unesco (2014), o ideal é que a tecnologia e a educação evoluam lado a lado com as necessidades educacionais.

Faz necessário ponderar sobre as limitações deste estudo, como por exemplo a restrição a somente algumas bases de dados, podendo ocorrer que algum estudo potencialmente relevante não tenha feito parte da amostra avaliada. Assim, sugere-se novos estudos envolvendo outros fatores e amostras e definindo parâmetros diferentes de inclusão e exclusão dos trabalhos, para a confirmação dos resultados encontrados.

\section{Referências Bibliográficas}

BARROS, D. M. V. et al. Educação e tecnologias: reflexão, inovação e práticas. Lisboa: s.n, 2011.

BATCHELOR, J.; HERSELMAN, M.; TRAXLER, J. Teachingand learning with new technology: a tough nut to crack. IST-Africa 2010, vol. 1, no. 7, pp.1-7, 19-21 May 2010.

BEDI, K. Tablet PC \& smartphone uses in education (Tablet Tours). In: 37th International Convention on Information and Communication Technology, Electronics and Microelectronics (MIPRO). p. 940-945, 2014.

BELAND, L.; MURPHY, R. III Communication: Technology, Distraction \& Student Performance. 2015.

BOLL, C. I.; LOPES, R. C.; LUCHINI, N. A. Tecnologias móveis e educação a distância: mais do que criar aplicativos é preciso saber o que fazer com eles. Simpósio Internacional de Educação a Distância e Encontro de Pesquisadores em Educação a Distância - SIED: ENPED:2016, p. 01-11, 2016.

CASTELLS, M. A galáxia da Internet: reflexões sobre a internet, os negócios e a sociedade. Rio de Janeiro: Zahar, 2003.

CORDEIRO, S. F. N.; BONILLA, M. H, S. Tecnologias digitais móveis: reterritorialização dos cotidianos escolares. Educar em Revista, Curitiba, n. 56, p. 259-275, 2015.

COSTA, E.; SILVA, A. P.; CORDEIRO, B. M. P.; SILVA, C. A. As tecnologias digitais chegaram! O que fazer? Formas inovadoras de aprender. In: DANTAS, L. G.; MACHADO, M. J. (Org.). Tecnologias e educação: perspectivas para a gestão, conhecimento e prática docente. 2 ed. São Paulo: FTD, 2014

FEENBERG, A. A fábrica ou a cidade: qual o modelo de educação a distância via web? In: NEDER, Ricardo T. A teoria crítica de Andrew Feenberg: racionalização democrática, poder e tecnologia. Ricardo T. Neder (org.). Brasília: Observatório do Movimento pela Tecnologia Social na América Latina/CDS/UnB/Capes, p. 153-175, 2010.

FEITOSA, T.; MACHADO, L. Escola institui tolerância zero para celulares na sala de aula. Gazeta Online. 26 ago. 2014.

GADOTTI, Moacir. Perspectivas atuais da educação. São Paulo em Perspectiva, v. 14, n. 2, p. 3-11, 2000.

GONÇALVES, H. A.; NASCIMENTO, M. B. C.; NASCIMENTO, K. C. S. Revisão Sistemática e Metanálise: níveis de evidência e validade científica. Revista Eletrônica Debates em Educação Científica e Tecnológica, v. 5, p. 193-211, 2015. 
GUENAGA, M. et al. Smartphones and teenagers, threat or opportunity. In: 15th International Conference on Interactive Collaborative Learning, IEEE, p. 1-5, 2012.

KNOBEL, M.; LANKSHEAR, C. A New Literacies Sampler. New York: Peter Lang. 2007.

LEE, V.; SCHNEIDER, H.; SCHELL, R. Mobile applications: architecture, design, and development. Prentice Hall, 2004.

LEMOS, A.; JOSGRILBERG, F. Comunicação e Mobilidade. Aspectos Socioculturais das Tecnologias Móveis no Brasil. Salvador: Edufba. 2009.

LUCENA, S. Culturas digitais e tecnologias móveis na educação. Educar em Revista, Curitiba, Brasil, n. 59, p. 277-290, 2016.

MARÇAL, E.; ANDRADE, R.; RIOS, R. Aprendizagem utilizando dispositivos móveis com sistemas de realidade virtual. CINTED-UFRGS, v. 3, n. 1, Porto Alegre: Maio, 2005.

MERIJE, W. Mobimento: educação e comunicação mobile. São Paulo: Peirópolis, 2012.

MORAN, J. Novas Tecnologias e o Reencantamento do Mundo. Revista Tecnologia Educacional. Brasil, vol. 23, n.126, p. 24-26, 1995.

MOURA, A. Geração Móvel: um ambiente de aprendizagem suportado por tecnologias móveis para a "Geração Polegar", Ambientes Emergentes, VI Conferência Internacional de TIC na Educação, p. 49-77, 2009.

MÜLBERT, A. L.; PEREIRA, A. T. C. Um panorama da pesquisa sobre aprendizagem móvel (m-learning). In: Associação Brasileira de Pesquisadores em Cibercultura, 2011, Florianópolis.V Simpósio Nacional da ABCiber.

NYIRI, K. Towards a philosophy of m-Learning. In: IEEE International Workshop On Wireless And Mobile Technologies In Education - Wmte, 2002.

ORGANIZAÇÃO DAS NAÇÕES UNIDAS PARA A EDUCAÇÃO, A CIÊNCIA E A CULTURA. Diretrizes de políticas da UNESCO para a aprendizagem móvel. Brasília: UNESCO, 2014.

POMPEO, Carolina. Professores disputam atenção de alunos com redes sociais. Gazeta do Povo. Londrina, 24 mai. 2014.

REIS, R. S.; LEITE, B. S.; LEÃO, M. B. C. Apropriação das Tecnologias da Informação e Comunicação no ensino de ciências: uma revisão sistemática da última década (2007-2016). RENOTE - Revista Novas Tecnologias na Educação, V. 15 № 2, 2017.

ROYLE, K.; STAGER, S.; TRAXLER, J. Teacher development with mobiles: Comparative critical factors. Prospects, v. 44, n. 1, p. 29-42, 2014.

SAMPAIO, R.; MANCINI, M. Estudos de revisão sistemática: um guia para síntese criteriosa da evidência científica. Brazilian Journal of Physical Therapy, scielo, v. 11, p. 83-89, 2007.

SANTA CATARINA. Lei n. 14.363, de 25 de janeiro de 2008. Dispõe sobre a proibição do uso de telefone celular nas escolas estaduais do Estado de Santa Catarina. Santa Catarina: Florianópolis, 25 mar. 2008.

SANTANA, R. C. M. et al. O uso de tecnologias móveis no ensino de ciências: uma experiência sobre o estudo dos ecossistemas costeiros da mata atlântica sul capixaba. Revista Ibero-Americana de Estudos em Educação, Araraquara, v. 11, n. 4 p. 2234-2244, 2016.

SENA, D; BURGOS, T. O computador e o telefone celular no processo ensino-aprendizagem da educação física escolar. In: $3^{\circ}$ Simpósio Hipertexto e Tecnologias na Educação, 2010, Pernambuco. Anais Simpósio Hipertexto.

TAPSCOTT, D. A hora da geração digital: como os jovens que cresceram usando a internet estão mudando tudo, das empresas aos governos. Trad. Marcello Lino. Rio de Janeiro: Agir Negócios, 2010.

UNESCO. O Futuro da aprendizagem móvel: implicações para planejadores e gestores de políticas. Brasília: UNESCO, 2014.

VALENTE, J. A. O Uso Inteligente do Computador na Educação. NIED, UNICAMP, 2012.

WAINS, S. I.; MAHMOOD, W. Integrating m-learning with e-learning. 9th ACM SIGITE Conference on Information Technology Education, Cincinnati, USA, pp. 31-38, 2008. 